\title{
Ascariasis intestinal. Tres casos pediátricos con complicaciones severas en Cauca, Colombia
}

\author{
Intestinal ascariasis. Three pediatric cases with severe \\ complications in Cauca, Colombia
}

\author{
Marco F. Sierra-Zúñiga', Luis R. Vásquez-Arteaga², Andry Mera-Mamián³, \\ Frank B. Méndez-Jiménez ${ }^{4}$, Francisco J. Timaná-Imbachí4, \\ Tania A. Romero-Arcos ${ }^{4}$, Daniel A. Ortega-Delgado ${ }^{4}$, \\ Ginna L. Belalcázar-Hernández ${ }^{4}$, Henry Muñoz-Orozco ${ }^{4}$ \\ Tatiana Cardona-Narváez ${ }^{4}$
}

Resumen. Se presentan tres casos clínicos de niños atendidos en el Hospital Universitario San José de Popayán en Cauca, Colombia, quienes consultaron por una infección severa causada por Ascaris lumbricoides. La severidad de la infección tuvo como consecuencia en el primer caso una colangitis aguda, en el segundo caso una obstrucción intestinal con peritonitis generalizada, y en el tercer caso una perforación intestinal y choque séptico. El objetivo de mostrar estos casos es dar a conocer estas presentaciones clínicas poco frecuentes en el entorno médico. Además, se resalta la importancia del conocimiento sobre esta especie de parásito, que termina siendo un problema más de salud pública, no solo en nuestro país, sino en gran parte del mundo.

Palabras clave: Ascaris lumbricoides, ascariasis, parasitosis intestinal, Colombia.

Abstract. We report three clinical cases of children treated at the San José University Hospital in Cauca, Colombia, who consulted with a severe infection caused by Ascaris lumbricoides. The severity of the infection resulted in acute cholangitis in the first case, in intestinal obstruction with generalized peritonitis in the second case, and in intestinal perforation and septic shock in the third case. The objective of this report

\footnotetext{
${ }^{1}$ Médico Cirujano, Residente de Pediatría. Departamento de Pediatría, Facultad de Ciencias de la Salud, Universidad del Cauca. Popayán, Colombia. E-mail: mafisi.unicauca@gmail.com

${ }^{2}$ Biólogo, Químico, Magíster en Microbiología con énfasis en Parasitología. Especialista en Entomología, Especialista en Epidemiología, Director del Centro de Estudios en Microbiología y Parasitología CEMPA, Departamento de Medicina Interna, Facultad de Ciencias de la Salud, Universidad del Cauca. Popayán, Colombia.

${ }^{3}$ Fisioterapeuta, Magíster en Epidemiología. Estudiante de Doctorado en Epidemiología y Bioestadística. Grupo de Investigación Movimiento y Salud, Facultad de Fisioterapia, Universidad CES. Medellín, Colombia.

${ }^{4}$ Médicos Cirujanos. Facultad de Ciencias de la Salud, Universidad del Cauca. Popayán, Colombia. 
is to inform the medical community about these unusual clinical presentations. In addition, the importance of this parasite is highlighted as a public health concern, not only in our country but also globally.

Keywords: Ascaris lumbricoides, ascariasis, intestinal parasitosis, Colombia.

\section{Introducción}

Las infecciones por parásitos intestinales son un problema de salud pública, en particular en los países en desarrollo, donde factores como las condiciones geográficas, la falta de higiene y de agua potable, la pobreza, el hacinamiento, y la desnutrición, entre otros, juegan un papel importante [1]. De acuerdo con la Organización Mundial de la Salud (OMS), se estima que alrededor de 3.500 millones de personas están infectadas [2,3], y que 880 millones de niños requieren tratamiento [4-6]. Los estudios epidemiológicos han mostrado prevalencias de infecciones parasitarias en las diferentes regiones a nivel global, que varían entre $30 \%$ y $83 \%$ [7].

En Colombia, según los datos disponibles de la Encuesta Nacional de Parasitismo Intestinal (ENPI 2012-2014), el $81 \%$ de los niños son afectados por parásitos intestinales, con una elevada tasa de morbilidad [8,9]. En particular, en el departamento del Cauca, en una población infantil indígena se encontró una prevalencia de parasitosis intestinal de 95,2\% [10], en tanto que en otra población en Tierralta, Córdoba, se encontró de 78,1\% [11]. Otro estudio llevado a cabo en 2004 por Agudelo-López y colaboradores [12], en un corregimiento del departamento de Bolívar, que incluyó población infantil y adulta, encontró una prevalencia general de infección de $92 \%$, sin diferencias significativas entre los grupos de edad. En contraste, en el área metropolitana de Barranquilla, Colombia, se encontró una prevalencia de $45,3 \%$ en niños, mucho menor a la encontrada en zonas rurales, lo que no sorprende por las condiciones más favorables en esta comunidad infantil urbana [13].

La ascariasis es una de las parasitosis intestinales más frecuentes en nuestro medio, a pesar de que los reportes muestran una aparente disminución en su prevalencia. De acuerdo con el estudio realizado por el Instituto Nacional de Salud de Colombia en el año 1980, la infección por Ascaris lumbricoides mostró ser la causa de parasitosis más frecuente, en el 38,6\% de los casos [14,15]. Más recientemente, en la Encuesta Nacional de Parasitismo Intestinal en Población Escolar 2012-2014, la prevalencia de ascariasis en los niños fue de 11,3\% [8]. A nivel mundial, para 2010, se calculó que alrededor de 820 millones de personas estaban infectadas con este parásito, mostrando una prevalencia a nivel global de 14,5\% [16], cifra que ya para el año 2019, ha aumentado a 1.500 millones de personas [17].

Los pacientes con ascariasis presentan síntomas que dependen de la fase del ciclo de vida en la que se encuentre el parásito y de su ubicación anatómica, al igual que de la intensidad de la infección, y del estado nutricional e inmune del hospedero. Las infecciones con parásitos adultos son usualmente asintomáticas, pero pueden manifestarse síntomas inespecíficos, como dolor abdominal, náusea, vómito y diarrea, y en los casos 
más severos pueden causar obstrucción intestinal y otras complicaciones [18].

A continuación, se presentan tres casos clínicos de pacientes que acudieron al Hospital Universitario San José, ubicado en el municipio de Popayán, Cauca, durante el año 2015, los cuales presentaban ascariasis con lesiones severas, y características particulares relacionadas, posiblemente, con la falta de intervención oportuna en promoción y prevención. El presente artículo pretende describir estos casos, dado el riesgo que genera la falta de medidas de higiene y la detección tardía de la infección, además de las consecuencias futuras relacionadas con el crecimiento y desarrollo de la población pediátrica [19].

\section{Presentación de casos}

\section{Caso clínico 1}

Paciente masculino de 6 años de edad, procedente del municipio de Caldono, Cauca, con cuadro clínico de 3 días de evolución de dolor abdominal intenso tipo cólico, sobre hipocondrio derecho, asociado a acolia y coluria, además de astenia, adinamia, hiporexia e ictericia. Sin alzas térmicas asociadas. Remiten desde centro de nivel uno con los siguientes paraclínicos: hemoglobina $12,4 \mathrm{mg} / \mathrm{dL}$; hematocrito 36,9\%; leucocitos 12.400 células $/ \mathrm{mm}^{3}$; neutrófilos $87 \%$; plaquetas 328.000 células $/ \mathrm{mm}^{3}$, bilirrubina total $1,98 \mathrm{mg} / \mathrm{dL}$, bilirrubina directa $1,8 \mathrm{mg} /$ $\mathrm{dL}$, bilirrubina indirecta $1,18 \mathrm{mg} / \mathrm{dL}$. Antecedente de consumo de agua no potable. Al examen físico se encontró con los siguientes signos vitales: presión arterial 118/78 mmHg; frecuencia cardíaca 113 latidos/minuto; frecuencia respiratoria 23 respiraciones/minuto; temperatura $36^{\circ} \mathrm{C}$; saturación de oxígeno $\left(\mathrm{SpO}_{2}\right) 97 \%$. Datos antropométricos: peso $24 \mathrm{~kg}$; talla $125 \mathrm{~cm}$; índice de masa corporal (IMC)
$15,36 \mathrm{~kg} / \mathrm{m}^{2}$. Valoración nutricional según parámetros de la OMS: talla para la edad $(T / E)=+2$ desviaciones estándar $(\mathrm{DE}) ; \mathrm{IMC}=0 \mathrm{DE}$.

El resto del examen físico mostraba un paciente álgido, con ictericia generalizada en piel y mucosas, con dolor a la palpación en hipocondrio derecho. Se solicitó una ecografía abdominal: "Ascaris lumbricoides en colédoco con neumobilia y signos indirectos de colangitis derecha" (figura 1). Se inició manejo antiparasitario. Se solicitó ecografía de control, la cual mostraba el parásito en vías intra y extra hepáticas. La colangiopancreatografía retrograda endoscópica (CPRE) reportó una papila con un parásito vivo, una vía biliar intra y extra hepática con múltiples parásitos vivos, que se extrajeron sin complicaciones aparentes. Su evolución clínica fue favorable posterior al manejo instaurado.

\section{Caso clínico 2}

Paciente femenina de 3 años de edad, procedente de la vereda La Cruz, en Inzá, departamento del Cauca. Cuadro clínico de 4 días de dolor abdominal tipo cólico, generalizado, de moderada intensidad, con periodos de exacerbación cortos, asociado con múltiples eventos eméticos y diarreicos, alza térmica subjetiva y distensión abdominal. Se remite desde centro de nivel uno. Al examen físico se encontraba con los siguientes signos vitales: presión arterial 96/71 $\mathrm{mmHg}$; frecuencia cardíaca 123 latidos/minuto; frecuencia respiratoria 32 respiraciones/ minuto; temperatura $36^{\circ} \mathrm{C} ; \mathrm{SpO}_{2} 94 \%$. Datos antropométricos: peso $13 \mathrm{~kg}$; talla $95 \mathrm{~cm}$; IMC $14 \mathrm{~kg} / \mathrm{m}^{2}$. Valoración nutricional según parámetros de la OMS: T/ $E=$ entre 0 y $-1 D E ; P / T=-1 D E ; I M C=-1$ DE. El resto del examen físico mostraba una paciente irritable, álgida, con mucosas pálidas y secas. Tinte ictérico genera- 

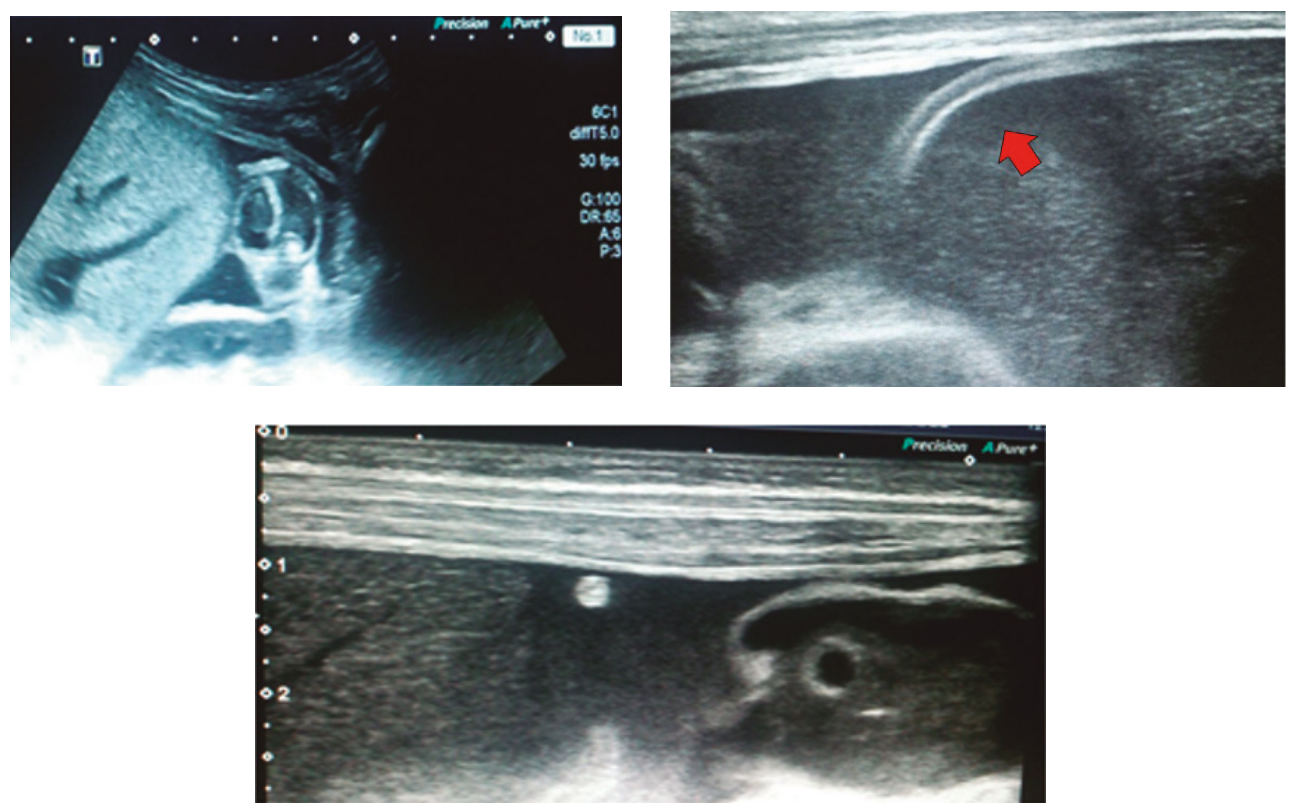

Figura 1. Ecografía abdominal con presencia de Ascaris lumbricoides en colédoco.

lizado, abdomen distendido, doloroso a la palpación, con masa a nivel de marco cólico, sin signos de irritación peritoneal en el momento de la valoración. Antecedentes de expulsión de A. lumbricoides. Se solicitó una radiografía de abdomen que mostró signos de "miga de pan", distensión de asas y ausencia de gas distal (figura 2). La paciente fue valorada por cirugía pediátrica por evolución no favorable, y se llevó a laparotomía, donde se encontró obstrucción intestinal por ovillo de los parásitos. Se consideró peritonitis generalizada con necesidad de cuidado intensivo por proceso séptico secundario. Su evolución clínica fue favorable posterior al manejo por cirugía pediátrica.

\section{Caso clínico 3}

Paciente masculino de 18 meses de edad, procedente de la vereda el Chimborazo en Morales, Cauca. Cuadro clínico de 8 días de diarrea, aso- ciado a alza térmica subjetiva. Por aumento en trabajo respiratorio consultó a centro de nivel uno, de donde fue remitido. Usuarios de acueducto de vereda, y deposiciones en letrina. Al examen físico se encontró con los siguientes signos vitales: presión arterial $77 / 49 \mathrm{mmHg}$; frecuencia cardíaca 155 latidos/minuto; frecuencia respiratoria 64 respiraciones/minuto; temperatura $37^{\circ} \mathrm{C} ; \mathrm{SpO}_{2} 96 \%$. Datos antropométricos: peso $9 \mathrm{~kg}$; talla 77 $\mathrm{cm}$; IMC $14 \mathrm{~kg} / \mathrm{m}^{2}$. Valoración nutricional según parámetros de la $\mathrm{OMS}: \mathrm{P} / \mathrm{E}=$ entre -1 y $-2 \mathrm{DE} ; \mathrm{T} / \mathrm{E}=-2 \mathrm{DE} ; \mathrm{P} / \mathrm{T}=-1$ $D E ; I M C=$ entre 0 y -1 DE. Se observó un paciente en malas condiciones generales, lucía tóxico a su ingreso con signos de deshidratación grado III, con tendencia a la somnolencia, además de signos de dificultad respiratoria. En la región abdominal presentaba distensión leve a moderada, dolor generalizado a la palpación, sin signos iniciales de irritación peritoneal según los datos de la historia clínica de ingre- 


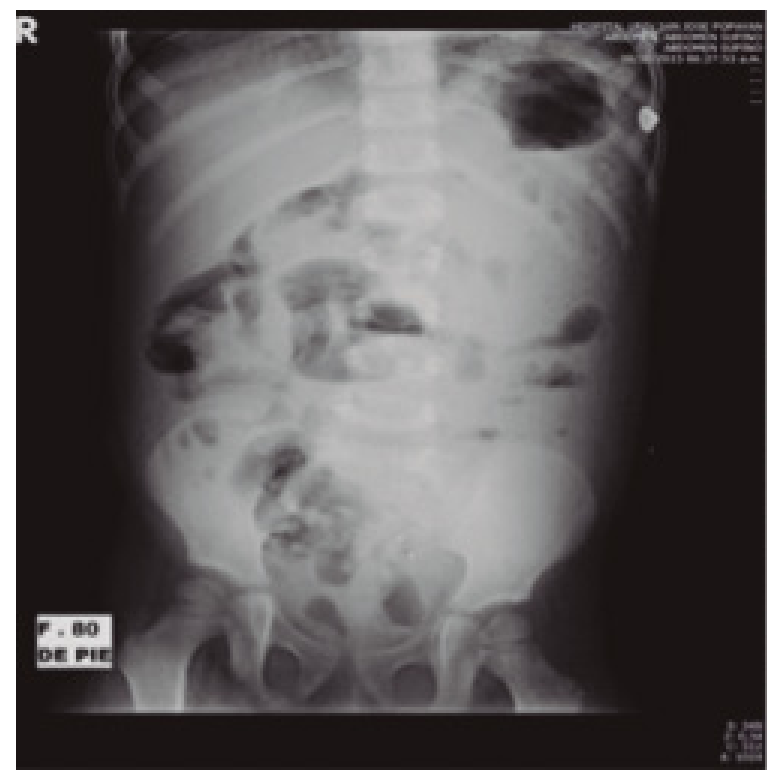

Figura 2. Radiografía de abdomen con signos de "miga de pan", distensión de asas y ausencia de gas distal.

so. Se solicitó una radiografía de abdomen debido a la alta sospecha clínica de una obstrucción intestinal por A. lumbricoides o un íleo secundario a sepsis (figura $\mathbf{3}$ ). Se documentó una obstrucción intestinal. Se dio manejo inicial con evolución tórpida. En controles radiológicos se evidenció presencia de neumoperitoneo. Finalmente, posterior a su estabilización inicial, el paciente fue llevado a laparotomía, donde se encontró peritonitis generalizada con parásitos en mesocolon, además de otro parásito libre en cavidad. Su evolución clínica fue favorable posterior al manejo quirúrgico.

\section{Discusión}

Este artículo describe los casos de tres pacientes pediátricos atendidos en el Hospital Universitario San José en el municipio de Popayán, Cauca, en el segundo semestre de 2015, con com- plicaciones derivadas por infección con A. lumbricoides. La severidad de la infección tuvo como consecuencia en el primer caso una colangitis aguda, en el segundo caso una obstrucción intestinal con peritonitis generalizada, y en el último, una perforación intestinal y choque séptico. Todos los pacientes mostraron evolución clínica favorable posterior al tratamiento.

Desde el punto de vista etiológico, las parasitosis intestinales pueden ser producidas por protozoos y por helmintos, ya sean nemátodos, tremátodos o céstodos. A. lumbricoides es un nemátodo que causa una infección muy frecuente, particularmente en áreas tropicales y países en desarrollo, como el nuestro [8]. El ser humano adquiere el parásito mediante la ingestión de huevos larvados del suelo contaminado con materia fecal. Las larvas eclosionan de los huevos una vez son ingeridas por el hospedero, y atravie- 


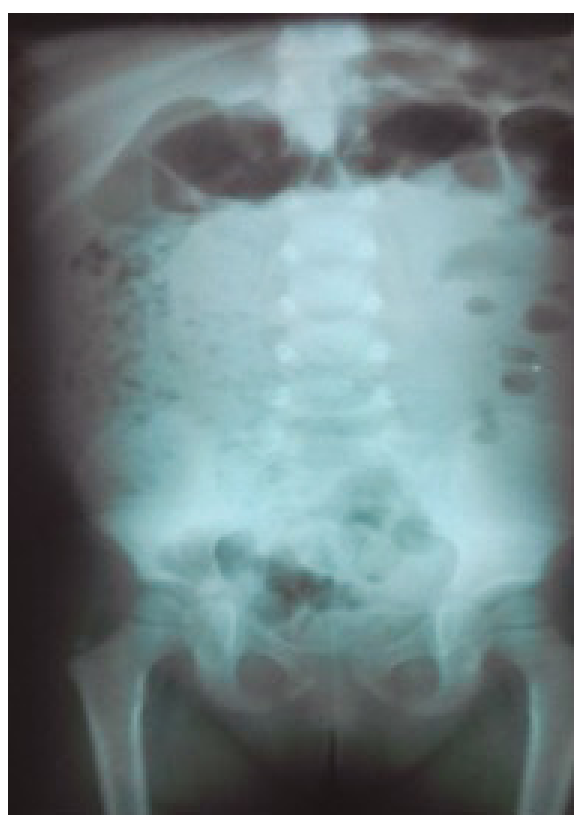

Figura 3. Radiografía de abdomen con signos de "miga de pan", distensión de asas, ausencia de gas distal, con sospecha de obstrucción por Ascaris lumbricoides.

san la mucosa intestinal, alcanzando la circulación portal para llegar al hígado y luego a pulmones, invadiendo los bronquios y alvéolos, donde sufren un periodo de maduración. Posteriormente, se desplazan a la faringe para ser deglutidas y reaparecer en el intestino delgado donde se transforman en adultos. Desde allí excretan huevos que salen en las heces, completándose el ciclo de vida. Tras su muerte, se expulsan espontáneamente [18,20].

Es importante destacar que las manifestaciones clínicas están relacionadas con el número de parásitos en el organismo y con la fase del ciclo en la cual se encuentren: fase pulmonar o fase intestinal. Clínicamente, la fase pulmonar de la infección puede pasar desapercibida; sin embargo, dependiendo de la intensidad de la infección y la exposición previa a alergenos larvarios, se puede hacer evidente el síndrome de Loeffler, el cual se puede presentar con fiebre, tos seca, disnea, hemoptisis e infiltrados pulmonares, y en algunas personas puede ocasionar reacciones alérgicas, como urticaria y asma [1,18]. En un estudio realizado por Hagel y colaboradores, en donde se pretendía determinar las posibles asociaciones entre el desarrollo de hiperreactividad bronquial y la respuesta inmunológica frente a $A$. lumbricoides, y en donde se evaluaron 470 niños de las escuelas de las comunidades rurales y urbanas en Venezuela, se observó clara evidencia de que la respuesta de la inmunoglobulina $E$ (lgE) frente a la infección, podía estar implicada en el desarrollo de la hiperreactividad bronquial en esta población [21].

Por otra parte, en la fase intestinal, además de las manifestaciones clínicas ya mencionadas, la mayoría de las complicaciones son debidas al desarrollo del parásito en el tubo digestivo, lo cual puede ocasionar obstrucción intestinal mecánica, obstrucción biliar, pancreati- 
tis, invaginación, apendicitis y granulomas viscerales, entre otras [22-24], como en algunos de los casos presentados en este artículo. A pesar de que el coprológico es la principal ayuda diagnóstica en los casos de parasitosis intestinal, el cuadro clínico de abdomen agudo que presentaron los tres pacientes en la consulta inicial, direccionaron el abordaje a estudios de imagenología, en los cuales se encontró obstrucción intestinal, con niveles hidroaéreos, ausencia de gas distal, y característicamente la presencia de una imagen denominada patrón en "madeja de estambres o miga de pan". En la ecografía abdominal se observó una estructura ecogénica curva, alargada y móvil, que correspondía al parásito.

La población infantil continúa siendo la más afectada con esta problemática, no solo por las lesiones directas de la infección, sino también a futuro, viéndose afectado su crecimiento y desarrollo [19]. La OMS recomienda tratar a todos los niños en edad escolar, a intervalos regulares, con antiparasitarios en zonas donde la infección por helmintos es común [25]. Sin embargo, al respecto, en una revisión Cochrane llevada a cabo en 2015, en la cual se analizaron 45 ensayos clínicos con más de un millón de niños, se llegó a la conclusión de que el tratamiento profiláctico podría tener un efecto en la ganancia de peso en aquellos infectados, pero que no había evidencia en cuanto a una mejoría del estado nutricional, la anemia, la cognición ni en las tasas de mortalidad [26]. En contraste, en un metaanálisis, cuyo objetivo fue establecer cuantitativamente la relación entre el acceso a agua potable, medidas de saneamiento e higiene, y la infección por helmintos transmitidos por el suelo, se encontró que el uso de agua tratada y las medidas de higiene básicas, como el lavado de manos antes de comer y después de defecar, el uso de jabón y el uso de zapatos, se asocian con una menor probabilidad de infección por helmintos transmitidos por suelo, logrando una reducción de al menos 33\% en las tasas de infección, con la implementación de cualquiera de esos parámetros, de forma independiente [27]. Lo que pone en manifiesto la importancia de las condiciones socioeconómicas y las medidas higiénico-sanitarias, y no solo las quimioprofilácticas en esta infección.

\section{Conclusiones}

Con alrededor de 1.500 millones de personas infectadas por $A$. lumbricoides a nivel mundial, esta parasitosis intestinal continúa siendo un problema importante de salud pública. Describimos tres casos con complicaciones severas y poco usuales, tratando de hacer un llamado al enfoque preventivo que se debe hacer sobre este tipo de patologías. Medidas de higiene básicas, como el lavado de manos antes de comer y después de defecar, el uso de jabón y el uso de zapatos, parecen ser medidas con un grado de protección mayor que las sugeridas actualmente por la OMS, como son la administración de antiparasitarios de forma periódica.

\section{Aspectos éticos}

Para la publicación de estos casos clínicos y el uso de las imágenes diagnósticas, se obtuvo consentimiento informado de los cuidadores de los menores, quienes autorizaron su divulgación con fines académicos.

\section{Agradecimientos}

A la Universidad del Cauca y al Departamento de Pediatría de la Universidad, en Popayán, Colombia. 


\section{Referencias}

1. Ojha SC, Jaide $C$, Jinawath $\mathbf{N}$, Rotjanapan $\mathbf{P}$, Baral P. Geohelminths: public health significance. J Infect Dev Ctries 2014;8:5-16. https://doi. org/10.3855/jidc.3183.

2. Navone GT, Zonta ML, Cociancic $P$, Garraza M, Gamboa MI, Giambelluca LA, et al. Crosssectional study of intestinal parasitosis in child populations in Argentina. Rev Panam Salud Publica 2017;41:e24.

3. Amer OS, Al-Malki ES, Waly MI, AlAgeel A, Lubbad MY. Prevalence of intestinal parasitic infections among patients of King Fahd Medical City in Riyadh region, Saudi Arabia: A 5-year retrospective study. J Parasitol Res 2018;2018:8076274. https://doi.org/10.1155/2018/8076274.

4. World Health Organization. Intestinal worms. Geneva: World Health Organization; 2018. Acceso 10 de septiembre de 2018. Disponible en https://www.who.int/intestinal_worms/more/en/.

5. Kucik CJ, Martin GL, Sortor BV. Common intestinal parasites. Am Fam Physician 2004;69:1161-1168.

6. López-Olmos J, Gasull J. Enterobius vermicularis (oxiuros) en la práctica ginecológica: clínica y citología. Experiencia de 3 casos. Clin Invest Ginecol Obstet 2011;38:197-201. https://doi. org/10.1016/j.gine.2010.01.009.

7. Cardona-Arias JA. Determinantes sociales del parasitismo intestinal, la desnutrición y la anemia: revisión sistemática. Rev Panam Salud Publica 2017;41:e143. https://doi.org/10.26633/ RPSP.2017.143.

8. Ministerio de Salud y Protección Social, Universidad de Antioquia. Encuesta Nacional de Parasitismo Intestinal en Población Escolar 20122014. Medellín: Facultad Nacional Salud Pública, Universidad de Antioquia; 2015. Acceso 15 de octubre de 2018. Disponible en https://www. minsalud.gov.co/sites/rid/Lists/BibliotecaDigital/ RIDE/VS/PP/ET/encuesta-nacional-de-parasitismo-2012-2014.pdf.

9. Ministerio de Salud y Proteccion Social. Atención integrada de la enfermedades prevalente en la primera infancia AIEPI. 2nd ed. Bogotá; Organización Panamericana de la Salud. 2015. p. 795.
10. Gaviria L, Soscue D, Campo-Polanco L, Cardona-Arias J, Galván Díaz A. Prevalencia de parasitosis intestinal, anemia y desnutrición en niños de un resguardo indígena Nasa, Cauca, Colombia, 2015. Rev Fac Nac Salud Pública 2017;35:390399. https://doi.org/10.17533/udea.rfnsp. v35n3a09.

11. Restrepo BN, Restrepo MT, Beltrán JC, Rodríguez M, Ramírez RE. Estado nutricional de niños y niñas indígenas de hasta seis años de edad en el resguardo Embera-Katío, Tierralta, Córdoba, Colombia. Biomédica 2006;26:517-527. https://doi. org/10.7705/biomedica.v26i4.317.

12. Agudelo-Lopez $S$, Gómez-Rodríguez L, Coronado $X$, Orozco A, Valencia-Gutierrez CA, Restrepo-Betancur LF, et al. Prevalencia de parasitosis intestinales y factores asociados en un corregimiento de la costa atlántica colombiana. Rev Salud Pública 2008;10:633-642.

13. Fillot M, Guzman J, Cantillo L, Gómez L, Sánchez-Majana L, Acosta BM, et al. Prevalencia de parásitos intestinales en niños del Área Metropolitana de Barranquilla, Colombia. Rev Cubana Med Trop; 2015. Acceso 9 de febrero de 2019. Disponible en http://revmedtropical.sld.cu/index. php/medtropical/article/view/93/94.

14. Manrique-Abril FG, Suescún-Carrero SH. Prevalencia de parasitismo intestinal y situación nutricional en escolares y adolescentes de Tunja. Rev CES Medicina 2011;25:20-30.

15. Cáceres E, Castaño de Romero L, Estupiñán $D$, López $M$, Páez $S$, Pinilla $C$, et al. In: Corredor A, Arciniegas E, Hernández C, eds. Parasitismo intestinal. Ministerio de Salud, Instituto Nacional de Salud. Santafé de Bogotá: Instituto Nacional de Salud; 2000. p. 67-68.

16. Pullan RL, Smith JL, Jasrasaria R, Brooker SJ. Global numbers of infection and disease burden of soil transmitted helminth infections in 2010. Parasit Vectors 2014;7:37. https://doi. org/10.1186/1756-3305-7-37.

17. World Health Organization. Soil-transmitted helminth infections. Geneva: World Health Organization; 2019. Acceso 20 de marzo de 2019. Disponible en https://www.who.int/news-room/ fact-sheets/detail/soil-transmitted-helminth-infections. 
18. Jourdan PM, Lamberton PHL, Fenwick A, Addiss DG. Soil-transmitted helminth infections. Lancet 2018;391:252-265. https://doi.org/10.1016/ S0140-6736(17)31930-X.

19. Banco Interamericano de Desarrollo (BID) , Organización Panamericana de la Salud (OPS), Instituto de Vacunas Sabin. Un Ilamado a la acción: Hacer frente a helmintos transmitidos por el suelo en América Latina y el Caribe. Washington: Pan American Health Organization; 2011. 35. Acceso 13 de noviembre de 2018. Disponible en https://www.paho.org/hq/dmdocuments/2011/ lac-report-esp-final-3-2011.pdf.

20. Dold C, Holland CV. Ascaris and ascariasis. Microbes Infect 2011;13:632-637. https://doi. org/10.1016/j.micinf.2010.09.012.

21. Hagel I, Cabrera M, Hurtado MA, Sanchez P, Puccio F, Di Prisco MC, et al. Infection by Ascaris lumbricoides and bronchial hyper reactivity: An outstanding association in Venezuelan school children from endemic areas. Acta Trop 2007;103:231-241. https://doi.org/10.1016/j.actatropica.2007.06.010.

22. Khuroo MS, Rather AA, Khuroo NS, Khuroo MS. Hepatobiliary and pancreatic ascariasis. World J Gastroenterol 2016;22:7507-7517. https://doi. org/10.3748/wjg.v22.i33.7507.

23. Das AK. Hepatic and biliary ascariasis. J Glob Infect Dis 2014;6:65-72. https://doi.org/10.4103/0974777X.132042.
24. Álvarez-Solís RM, Gutiérrez-Lucatero $S$, Vargas-Vallejo M. Diferencias clínicas entre oclusión y suboclusión intestinal por Ascaris lumbricoides. Datos que orientan al tratamiento quirúrgico. Acta Pediatr Mex 2011;32:156-162.

25. World Health Organization. Guideline: preventive chemotherapy to control soil-transmitted helminth infections in at-risk population groups. Geneva: World Health Organization; 2017. Acceso 25 de septiembre de 2018. Disponible en https://apps.who.int/iris/bitstream/handle/10665/258983/97892415501 16-eng.pdf; jsessionid=127653C192A6A9EB7244410B51A93E5B? sequence=1.

26. Taylor-Robinson DC, Maayan N, Soares-Weiser K, Donegan S, Garner P. Deworming drugs for soil-transmitted intestinal worms in children: Effects on nutritional indicators, haemoglobin, and school performance. Cochrane Database Syst Rev 2015:CD000371. https://doi. org/10.1002/14651858.CD000371.pub6.

27. Strunz EC, Addiss DG, Stocks ME, Ogden S, Utzinger J, Freeman MC. Water, sanitation, hygiene, and soil-transmitted helminth infection: A systematic review and meta-analysis. PLoS Med 2014;11:e1001620. https://doi.org/10.1371/journal.pmed.1001620. 